\title{
Recent Advances in Connected Vehicles via Information-Centric Networking
}

\author{
Z. Zhu*, J. Loo*, Y. Chen ${ }^{\dagger}$, K.K. Chai ${ }^{\dagger}$, T. Zhang* ${ }^{\dagger}$ \\ *School of Science and Technology, Middlesex University, London, UK \\ \{zz130@live, J.Loo@\}mdx.ac.uk \\ ${ }^{\dagger}$ School of Electronic Engineering and Computer Science, Queen Mary University of London, UK \\ \{yue.chen, michael.chai\}@qmul.ac.uk \\ ${ }^{*}$ School of Information and Communication Engineering, Beijing University of Posts and Telecommunications, China \\ zhangtiankui@bupt.edu.cn
}

Keywords: V2X, VANET, ICN.

\begin{abstract}
V2X communication technology allows vehicles to communicate with each other, infrastructures as well as other parties. It is considered as a vital role in realizing future Intelligent Transport System (ITS). On one hand V2X is facing various expectations that requested by different features of applications, On the other hand, V2X has to overcome problems caused by the natures of high mobile vehicle environment. ICN proposed as the a substitution for future Internet rely on its naming design is likely to associate with $\mathrm{V} 2 \mathrm{X}$ well in contrast to convention TCP/IP solution. This paper viewed recent relevant literatures from which unaddressed problems are identified with discussion of possible solutions. From this work, we are positioning our future efforts to fulfil such gaps.
\end{abstract}

\section{Introduction}

Vehicle-to-Everything (V2X) covers the communications between vehicle-to-vehicle, vehicle-to-infrastructure, vehicleto-pedestrian $[5,9,19]$ as illustrated in Figure 1 . This promising wireless technology enables vehicles to share diverse information such as their current speed, location with other vehicles in vicinity and remote infrastructures in practical complex traffic condition. V2X is considered as a vital role in realizing future Intelligent Transport System (ITS) [33] where different technologies are integrated to provide optimized traffic management, safety ensuring, mobility for traveling.

Although V2X technology greatly benefits to safety and travel efficiency, its applications raise new challenges at the same time. On one hand, V2X communication faces various expectations that requested by different features of applications. On the other hand, V2X has to overcome physical wireless problems caused by the highly mobile vehicle environment.

Conventional TCP/IP was designed for early Internet that handles a single conversation between two entities, cares about where data resides focusing on maintaining end-to-end connection between endpoints and centric hosts can hardly fit in V2X scenario where the network is primarily used as a tool for diverse contents dissemination instead of IP's inherent point-to-point communication. Furthermore, V2X is a new wireless paradigm with its content widely distributed over the network should no longer restricted by a single source provider but IP can only identify endpoints' address is not helpful for content distribution. IP also confines the mobility for the increasing wireless devices [51]. Thus, a novel approach with native support to improve such issues is desired.

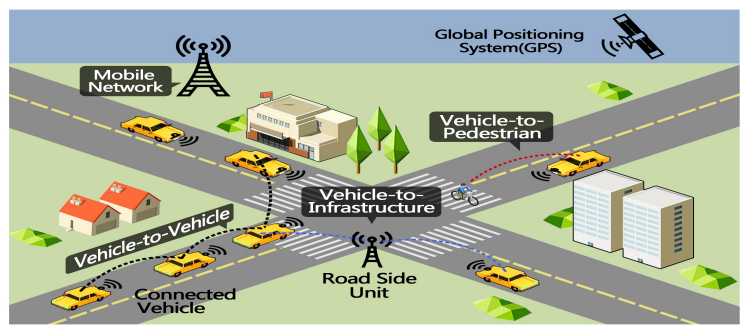

Figure 1: V2X communication

Content-Centric Networking (CCN) [40,41] as one of Information-Centric Networking (ICN) $[3,37]$ architecture proposed for future Internet is an ideal alternative since CCN cares about the data itself not its location thus removes IP address restriction. Data are hierarchically named directly to be transmitted instead of embedded into a conversation, so as to eliminating the obstacle of supporting mobility and enabling scalable efficient data dissemination with low traffic requirement by its in-network caching. Therefore, CCN copes well with V2X content distributed background.

However, it is not straightforward for CCN to adapt to vehicular environment. Several aspects have to be reconsidered to combine these two seamlessly. Recent research attentions have been paid to how data should be properly named to be identified and delivered, How and when data should be forwarded to the consumer in high dynamical topology caused unstable wireless connectivity with reference to CCN's data structure, A well-designed caching mechanism is expected to decide what data should be cached for satisfying future requests and how it should be replaced. Early works still left open issues to be pursued in following research.

Thus this paper is motived and the rest of paper is organized as follows: Section 2 describes vehicular communication and how ICN can benefit to it. Section 3 reviews related works followed by a discussion of possible solutions based on identified gaps in section 4. Lastly, a conclusion is made in section 5 . 


\section{Overview}

\subsection{VANET}

VANET has its mobile pattern of higher velocity, traveling path fixed and predictable. Based on Dedicated Short-Range Communication (DSRC)/Wireless Access in Vehicular Environment (WAVE) [165,32,45] as standards for PHY and MAC layers, VANET communication is able to archive high throuughput with low delay. Thus, a variety of applications covering different aspects have emerged [7,9,39].

Safety applications:emergency braking, lane change warning collision avoidance, hazard notification. Efficiency applications: congestion management, electronic toll collection, parking availability. Commercial applications: Internet access live video stream. Comfort applications: weather information, autonomous driving, journey time estimation.

However, VANET has its unique features to be taken into account:

High dynamic topology: This is caused by the high speed of vehicles and limit transmission range. Vehicles moving at $40 \mathrm{~km} / \mathrm{h}$ in urban and $100 \mathrm{~km} / \mathrm{h}$ on highway while radio coverage is only hundreds of meters which results in vehicles join and leave networks frequently thus network topology is constantly changing and partitioned [17].

High transmission and computation capability: Vehicles are equipped with on board devices and able to provide continues power in terms of computation and data storage that means hardwares issue are not constraints [9].

Unstable connectivity: The inherent wireless transmission natures enlarged by high dynamic vehicles. In such a context, connectivity is therefore frequent disconnected and intermittent, the link duration trends to be very short.

Large scale: Each vehicle acts as a node in the network. This means the entire network can expand to the degree of roads coverage that include a large number of participants.

Predicable mobility pattern: As vehicles have to follow roadways, that is to say the movement of nodes are limited to road layout. With refer to GPS system and road map nodes' future location can be predictable.

\subsection{ICN}

Initially, CCN was the running prototype under the Information-Centric Networking (ICN) research direction raised by Van Jacobson. ICN represents a general trend of future Internet architecture that evolves from the today's host centric, end-to-end, IP focused architecture to a content centric and distributed one. CCN and Named Date Networking(NDN) [24] are the typical instances of the broad ICN umbrella. This paper use CCN and NDN interchangeably.

CCN basically changes the definition of network service from transmitting data to a certain destination address to requesting data identified by a given name from potential providers. As CCN focus on the named data itself instead of where data resides, the identified name is the key for both Interest packets and Data packets (Figure 2) which are the two kinds of data in NDN. Interest packets containing the content name are generated by consumers for requesting desire content and this Interest packets disseminate in the network to be received by intermediate routers then routers figure out proper outgoing face to forward this Interest accordingly. Once, Interest hits the providers having corresponding data, they reply with Data packets that travel back to consumer following the reversed forwarding path left by the Interest packets. Meanwhile Data packets are cached at intermediate routers along the way for future reuse by potential consumer with same Interests [22].
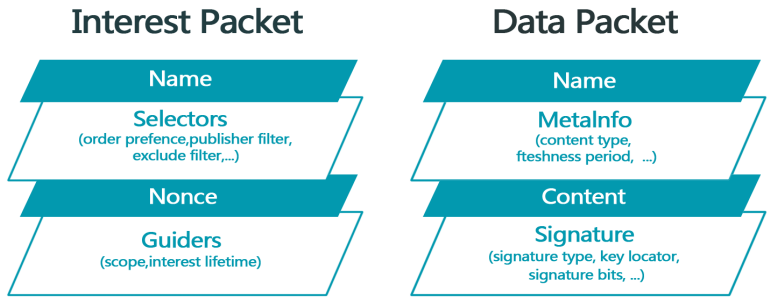

Figure 2: Packets of NDN

There are three components in each NDN router: Pending Interest Table (PIT) maintains a entry list of unsatisfied Interests that will be used for Data packets routing; Forwarding Information Base (FIB) keeps a list of faces where unsatisfied Interest should be forwarded to; Content Store (CS) caches Data packets arrived at this router for subsequent reuse [23]. Consumer sends out an Interest packet, it arrives at NDN router. Firstly, a lookup will be performed in CS to match the Interest. If a match is found, the router sends back the Date via the incoming face; If not, a further lookup acts in PIT. At this point, if a match is found, the interest is discarded and only the incoming face of the Interest is recorded in PIT; If not, a new entry of incoming face is created in PIT then router forwards the Interest to the potential providers according to the FIB. An Interest dose not match any of these tables will be dropped or trigger the node to discovery alternate routes. Figure 3 sketches above processes.

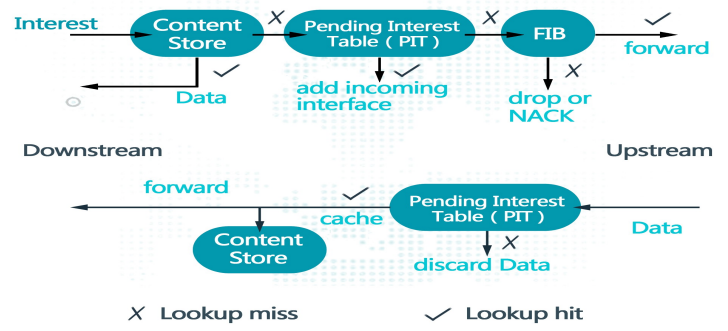

Figure 3: Forwarding in NDN

\subsection{CCN for VANET}

Although VANET plays an important role in V2X communication due to its high mobile and wireless natures, several challenges are obstructing its developing. This subsection argues how CCN can meet them thus is ideal to networking vehicular environment.

(i) Routing. Routing for VANET were mainly derived from Manet where the mobility pattern changes differently and topology varies slower. Therefore, existing routing protocols proposed for in Manet are rarely suitable for VANET [18]. Due to the vehicles are of high velocity, V2X has a highly dynamic network topology which contributes to an intermittent end to end connectivity with very short link duration and limited transmission range. In today's Internet, IP that is heavily 
relied on as a thin waist to associate with both upper and lower layers. Every communication can not avoid the IP address or topology location which is a major obstacle to support mobility.

While routing and forwarding in $\mathrm{CCN}$ are based on the name of content rather than the location of content, it tremendously improves mobility. Routers advertise name prefix of individual content across the network in help of building each router's FIB not conventional IP address. There is no IP addreess exhaustion and management issues. Furthermore, In CCN the global unique name of content always remaining the same regardless the speed or direction change of nodes which ensures communication in contrast to connectivity breaks problem or tunneling additional traffic via third party of IP. And the native support of multi-path routing greatly benefits to vehicular condition.

(ii) Scalability. Vehicle population growth leads to a large scale and dense vehicular communication environment. Designing such network should consider scenarios where condition changes between sparse to high dense. In addition, there is no such standard that unifies this network in a global manner, standards from different vehicle manufacturer are mismatch.

In CCN, it has an in-network caching mechanism at each routers. Each chunk of data is uniquely named that can be cached in CS for subsequent request. Although the CS is just buffer memory, with the naming system enables a same piece of data to be used for multiple times rather than IP routers which are not able to reuse it after forwarding done. That is to say, when network size increases, the in-network caching functionality can greatly reduce the traffic load from a centric host because contents are widely distributed as cached replicas across the network. VANET can benefit from such advance when vehicle number rises at urban or during peak hours.

(iii) QoS. A network guarantee less delay, minimum retransmission, stable connectivity is ideal for vehicular communication. One such will eventually enhance user experience regardless the blooming of application of all kinds. However, it is a challenging job for VANET designing [27].

NDN is operating on top of unreliable packets delivery. Different from today's architecture transport layer, NDN shifts transport protocols into applications, supporting libraries. To ensure reliable transmission, a consumer that is not satisfied within given timer will resend the interest packets. Congestion control is taken care by each NDN router , it manages the interest forwarding rate to balance network traffic load [39]. Caching functionality shorten the retransmission path from initial producer to previous hop where data lost which is idea for VANET because of the vehicular features so as to further avoid bandwidth being consumed by repeating transmission therefore it surely improves QoS in VANET.

\section{Recent work}

Early works $[13,24]$ explored the feasibility of CCN in conjunction with VANET, proved CCN indeed is a promising solution for V2X communication. As CCN was not initially proposed for a wireless mobile network, besides aforementioned VANET's features, CCN has to be well modified and essential extensions have to be made to seamlessly adapt to vehicular environment. we are attracted to undeveloped areas through a literature review (summarized in Table 1),

\subsection{Network architecture design}

As the benefits and advances of CCN design is gaining more and more attention, plus CCN addresses mobility issues in a native way, researching on such a network architecture is becoming popular.

[1,4] Proposed a hierarchical NDN architecture for V2X. By leveraging a complementary approach, LTE is used for communication between vehicles and infrastructures in low dense condition while DSRC is used for V2V, these works solve the problem cause by limited DSRC transmission range as well as poor caching performance since there is no route between produces and consumers in low vehicle density situation. Thus it significantly enhance the delivery performance and can be considered as a starting point for further routing researching.

[11] Provides a Internet content distribution based on NDN among vehicles. Although it provides intelligent response and breakpoint resume to improve VANET quality of experience, this work is based on a simple one hop large size content distribution mechanism in contrast of vehicular multiple hops communication.

[13,49,51] Point out defects of IP mobility then provide alternatives by utilizing NDN in vehicular networks for supporting mobility. Through such designs NDN can be concluded as an effective solution to handle connection disruption characterized by high dynamic networks.

[42] Introduces a Pub/Sub mechanism, extends NDN to both pull-based and event driven model for better data collection and dissemination to fits in V2X environment. In this design, each vehicle has to constantly broadcast message to be received by RSU for subscription it well addresses the low delay of time-sensitive safety application requirement. [36] also from application view that benefits from CCN developed a Traffic Violation Ticketing (TVT) system for traffic police to automatically issue tickets to offenders.

[25] Attention has been paid to content segmentation to ensure reliability. It provides a efficient selective interest retransmission by adding an extra field to interest enables consumers to reply what are missing then require retransmission. Furthermore, it sets up interest packets RTT as a reference for retransmission thus provide a well-designed yet reliable delivery in vehicular CCN.

Above works have taken pioneering steps to apply CCN into V2X domain. Their valuable evaluations conclude that CCN decouples content from conventional IP address to support mobility by naming innovation hence substantially benefits to vehicular communications as well as to be resilient to bear unstable connectivity via in-network caching. They can be regarded as strong motivations to exploit specific design, for instance, Scalability could be further considerate with more consumers and producers scenario in urban background, a better caching policy handing low content popularity could improve the performance and further thoughtful design ranging from naming scheme, forwarding strategy, security and privacy concerns lies ahead. 


\subsection{Caching}

Caching functionality of CCN decentralized content being confined in a centric host thus significantly reduces network traffic load. Various caching policies and caching replacement policies can be implemented at each caching point depending on specific requirements of applications.

$[8,14]$ Proposed a caching scheme that pre-distribute content. The probability of successful content retrieve is formulated by Integer Linear Programming problem under a V2I network where each APs is acting as caching point.

[20] Worked on caching point selection. A minimum vertex cover set algorithm is used to initialize node status then CCBSR decides the caching point with regards to VANET features. Results turned to be a faster content retrieving method, since it is based on video stream of which content piece could be fetched from multiple source resulting in an arrival disorder manner, it should associate with a proper caching play order mechanism.

[46] A proactive caching approach for NDN where unsatisfied content before a user handover will be cached close to it for subsequent retransmission. So the user can get the content immediately once connection reestablished. This approach avoid unnecessary retransmission hence shorten the content retrieve delay with low handover cost.

Despite these good caching works there are still enhancements can be made. One can take advantages of mobility model of vehicle where a more precise predication of future position of node could be made dependent on vehicle's speed and moving direction. Alternatively a more efficient caching strategy aiming to prevent storage from being wasted or under a limited content store size rather than simply caches each file at every node could be investigated.

\subsection{Naming}

CCN uses hierarchical naming design that replaces conventional IP based solution. Each content is uniquely named after itself and exists independently to be direct used so as to eliminates IP address related issues.

[50] Proposed a hierarchical naming policy for both efficient pull-based and push-based communication. In their desig$\mathrm{n}$, the fields of name structure include destination location, data types, source location, data provided by this consumer and the next location towards destination. It addressed the challenge of content aggregation and mobility management.

[21] Proposed a data naming structure as /traffic/geolocation/timestamp/data type/nonce where traffic represents the application id; geolocation consist of road id, direction, section number; time stamp uses UNIX time stamp format; data type suggest data itself. The use of data names allows traffic information to be disseminated among vehicles that are best suit in V2X communications.

Similarly, [6] embedded geographic areas information into data names by a encoding algorithm that convert the location of $\langle\mathrm{x}, \mathrm{y}\rangle$ coordinates into name /ndn/ucla/parking/.../cn. With results showing, this work not only improves cache hit ratio but also set specific application free to forward Interest and Data packets.

Furthermore, since naming in CCN requires a universal understanding that penetrates every aspect (e.g. routing, caching
, security) of whole system, it is still an open challenge to design unified yet thoughtful data name structures for vehicular communication ensuring subsequent developments for networking vehicle communication.

\subsection{Routing and Forwarding}

There is a lot of attention paid to this field but ones proposed for Manet are not suitable for vehicular CCN due to their different mobile patten and CCN routing mechanism. Recent works are mainly focusing on discovery content process for interest packets forwarding. In addition, vehicles are related to geographic location, many works exploit such information to reduce broadcast inefficiency.

[31] Source Selection Dynamically Network Coding-based Information Centric networking(SSDNC) protocol, taking the advantage of multiple available source by CCN to make forwarding decision according to weights on different interface.

[44] Each node additionally keeps a Cluster Common Interest Table. Vehicles are divided to dynamic and logical clusters based on their interest which leads to low complexity for content distribution and increase the scalability.

By utilizing physical location [11] Proposed a hybrid forwarding strategy (HVNDN) using opportunistic and probabilistic strategy associated with geographic information to address the problem that FIB lacks of decision results in Interest packets to be flooded across network causing broadcast stor$\mathrm{m}$. Hierarchical Bloom-Filter Routing [47] is a proactive geography-based content discovery scheme. Vehicles are partitioned by partitioning road map and Bloom-filters are used to advertise name prefix in each partition. it achieves much lower response time and less traffic. [12] it combines data names with producers' geographic areas. Interests are forwarded towards producers across the network by a shortest roads layout route. A content discovery and selection scheme are designed to pick the best source from several geographic locations.

CONET [34,35] from data point of view, it allows interest packets to count hops for the use of data packets delivery. Data packets are able to figure out the minimum hops when being forwarded towards original consumers.

[48] Last Encounter Content Routing (LER) reduces the content discovery overhead caused by flooding interest across network by keeping content location at each node and exchange this content list when nodes meet each other for updating.

Above works are commonly based on initial CCN proposal that for the Data packets forwarding part, they are supposed to follow the exact same path in a reversed order as Interest packets. It is a clever design but also misleads researchers for designing forwarding strategy in a wireless vehicle background. Data packets simply are not always being able to achieve such assumption if take a look at the V2X communication features in section 2.1.

\section{Future research direction}

Previous discussion proved that ICN significantly benefits to vehicular communication. As we are in early stage of research, it is still far from a seamless integration of these two technologies. We think twice of existing literatures that there are still research gaps expecting further dedication. By identi- 
Table 1: summary of related works

\begin{tabular}{|c|c|c|c|}
\hline category & reference & Purpose and summary & Open issue \\
\hline \multirow{5}{*}{$\begin{array}{c}\text { Network } \\
\text { architec- } \\
\text { ture }\end{array}$} & {$[1,4]$} & Hierarchical design for both V2V via DSRC and V2I via cellular communication & \multirow{5}{*}{$\begin{array}{l}\text { Scalability, } \\
\text { Security } \\
\text { consideration, } \\
\text { Broadcast bandwidth } \\
\text { wasting }\end{array}$} \\
\hline & [11] & Based on one hop large size content distribution design & \\
\hline & {$[13,49,51]$} & $\begin{array}{l}\text { Elaborates CCN is able to handle connection disruption well in contrast to IP } \\
\text { solution }\end{array}$ & \\
\hline & {$[36,42]$} & Pub/Sub mechanism for event driven application & \\
\hline & [25] & Content segmentation and redundancy free retransmission & \\
\hline \multirow{3}{*}{ Caching } & {$[8,14]$} & Pre-distribute content, ILP for calculating content retrieve ratio & \multirow{3}{*}{$\begin{array}{l}\text { Predicable mobility } \\
\text { model, } \\
\text { Storage wasting }\end{array}$} \\
\hline & [20] & CCBSR determines caching points in VANET & \\
\hline & [46] & PCNDN enables retransmission from immediate caching nodes & \\
\hline \multirow{2}{*}{ Naming } & {$[50]$} & Hierarchical naming policy for pull and push based communication & \multirow[t]{2}{*}{ Lacks unified design } \\
\hline & {$[6,21]$} & Embed geographic location into data name structure & \\
\hline \multirow{5}{*}{$\begin{array}{l}\text { Routing } \\
\text { and } \\
\text { forward- } \\
\text { ing }\end{array}$} & [31] & Forwarding decision based on weights of interfaces & \multirow{5}{*}{$\begin{array}{l}\text { Requires } \\
\text { consideration for Data } \\
\text { forwarding when no } \\
\text { previous connection } \\
\text { exists }\end{array}$} \\
\hline & [44] & Vehicle cluster based, each nodes keeps a CCI table & \\
\hline & {$[11,12,47]$} & Geographic location based approach for Interest forwarding & \\
\hline & {$[33,35]$} & Use hop counter to guide Data packets forwarding & \\
\hline & [48] & LER discovery content by exchanging information when vehicles meet & \\
\hline
\end{tabular}

fying such blank space and possible solutions discussion, we take them as guidelines to positioning our subsequent works as well as reinforcing ongoing literatures.

\subsection{Content discovery}

In CCN, names are the only identifiers for each content. Content will not be discovered unless the name identifier of requests match with content's. Content discovery should focus on acquire the name of available content for Interest to match. A consumer initiates content discovery mechanism by sending request towards publishers, upon receiving reply consumer then configure out what content is available so as to issue meaningful subsequent Interests accordingly. However, when apply to a high mobile instance, request has higher percentage of not being answered before timeout due to unstable and short duration connectivity leading to a content availability misjudge. Furthermore, when comes to dense nodes condition, each node broadcasts request increases network load. One can also take advantage of that enable sharing of content availability information among nodes self. Therefore opens a direction for further pursuing such method in CCN-VANET system.

\subsection{Source selection}

CCN-VANET provides consumer multiple location of Data source and a node is connected to potential source via different face. Source selection can be treat as selection of best face links to source at minimum cost. The metric for such cost can be transmission delay, connection reliability, transmission distance etc. In CCN-VANET, we can measure the retransmission times of Interest packets to ensure selected source provides the best reliability. The reason being is that CCN no longer has a conventional transport layer like today's Internet, it moves the functions of today's transport protocols up into applications, their supporting libraries, and the strategy component in the forwarding plane [41]. In order to achieve reliable delivery, the retransmission is taken care of by final consumer. If no desire Data packets are received within reasonable period of time, the final consumer is responsible for reissue the Interest packets. By comparing the retransmitted Interest counter through different faces at each node as showing in figure 4 [28], decision can be made for source selection depend on transmission reliability.

\subsection{Data packets forwarding}

In initial CCN design, Data packets are expected to traverse

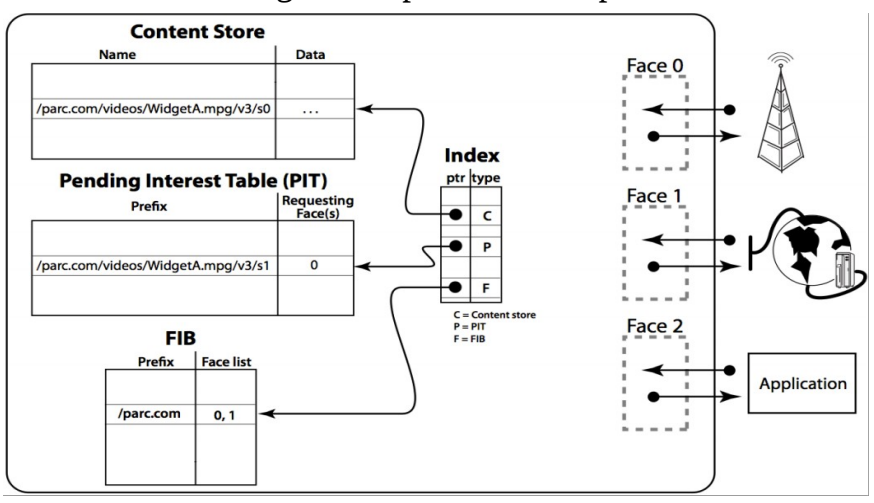

Figure 4: NDN node

back to the initial consumer following the exact same path as Interest packets. However in vehicular communication due to high dynamic topology changing and wireless features, we argue this common agreement upon which above works assumed is untenable. Any disconnection which is commonly appears in VANET between arbitrary adjacent hops in which Interests have been through means a breakage in Data forwarding process. As figure 5 shows, desire Data are available at both repository and cached node (V5), green circle represents transmission range, blue line represents estatablished connection, red line means disconnection. In figure 5 (1), consumer V1 sends Interest eventually hits avail-able providers via V1-V2-V4-repository and V1-V2-V3-V5. For Data forwarding (figure 5 (2)), according to aforementioned works, obviously Data can not be received by V1 since V2 plays an crucial intermediate node but at this instance it is disconnected. In fact, the closer Data are getting to consumer the higher probability it would suffer above phenomenon. To our best knowledge, no such works have addressed this issue, hence, we are motivated to explore a suitable forwarding scheme exclusively works for Data packets with reference to CCN definition and V2X communication.

Solutions to support Data packets forwarding can be learned from existing VANET routing protocols that are either topology-based or geo-location based (summarized in table 2). 
Table 2: VANET routing

\begin{tabular}{|c|l|l|l|l|}
\hline Protocol & \multicolumn{1}{|c|}{ Type } & \multicolumn{1}{c|}{ Overhead } & \multicolumn{1}{c|}{ Pros } & \multicolumn{1}{c|}{ Cons } \\
\hline FSR[2] & Topology-based (proactive) & All link states & Low latency, reduce broadcast overhead & Bandwidth waste \\
\hline AODV[30] & Topology-based (reactive) & Path states & Reduce network burden & Require discovery \\
\hline GPCR[18] & Geo-location-based & Beacon & No link state or connectivity maintenance required & Stable connectivity \\
\hline
\end{tabular}

From topology-based view, proactive link-state routing requires each node maintains the knowledge of universal connectivity of the network, a node's routing table then made from a collection of the best logical path from itself to every possible destination. While reactive routing opens a route only when communication is on demand. It has a route discovery phase where query packets are flooded into network until a path is found and only maintained during the communication.

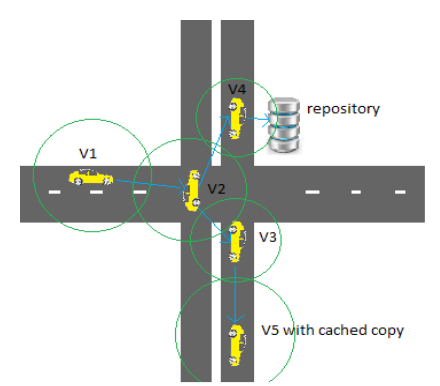

(1) Interest forwarding

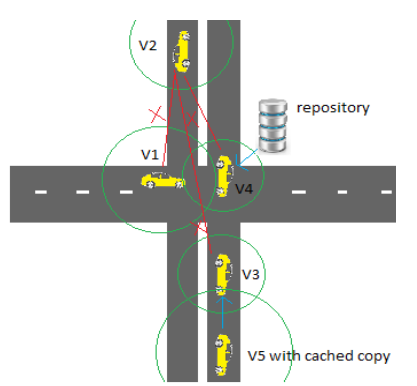

(2) Data forwarding
In geo-location based approach, forwarding decision is determined by geographic location of destination and node's onehop neighbour. It assumes each node knows its location by GPS. The location of destination is carried in the header of packets generated by source and the location of neighbours are obtained from beacons message. Geo-locaton based routing does not need to exchange link state information or maintain exist routes like topology-based routing does.

Our proposal still complies with CCN design where during Data packets forwarding (figure 3), it goes through a multihop basis guided by PIT entry at each node. Whenever situation as figure 5 (2) occurs it means the node about to forward Data is disconnected with the next downstream node as PIT entry indicates. At this point, an AODV alike route discovery phase is triggered in search of a new route. A query RREQ containing Data name prefix is broadcast into network. Each intermediate node performs a procedure to record its previous hop. Eventually, RREQ arrives at a node where name prefix find a match indicating the original consumer that asked for the Data packets has been found. Next a reply RREP then travels back to route request node according to the hop information collected from backward learning. At each node of the path the relevant PIT is updated by the faces in the order of what RREP has been through. When RREP comes back to route request node, a new chain of PIT entries is expected to constructed. At this point, the expired previous multihop path is replaced by the new one thus Data packets are able to be transmitted back to original consumer.

\subsection{Multiple source routing}

FIB of CCN for each name prefix has more than one outgoing faces thus allows a parallel query for multiple sources data. Typically in CCN-VANET, besides the permanent source providers each node could be a potential source provider that cached a replicate. With regards to VANET dynamical topology changing and node density varying, there is a high possibility that the path to the original source can not be maintained at all times. From section 3.4, existing designs [47] may copes well with unsharable data, achieves much lower response time and less traffic but only use a single path with a single content source yet introduces more overhead. [31] provides a good throughput and short delay performance based on 15 nodes in a $2 \mathrm{~km}$ with 3 lanes road condition which did not take scalability into consideration. Given a limit transmission range when it comes to low dense node condition the data propagation speed drops while in high dense condition most works are rely on a broadcast either to build FIB or to frequently exchange source location of neighbour information, contributing to large overhead and bandwidth wasting yet introduce additional list management. Future work should continue on exploiting a design performs on content forwarding well simultaneously address impact caused by VANET characteristics.

\subsection{Cross-layer design}

Include cross-layer design in ICN-based V2X can maintain the function of original layer but also brings in interactivity to different layers and information can be shared across layer boundaries to achieve specified QoS [29]. Cross-layer can be information flow within interfaces; merging layer functions; collaboration across different layers. In CCN-VANET, physical layers can provide transmission power information, link quality information can be extracted from MAC layer, vehicles' position and moving patterns can be obtained from application layer for predication. By utilizing such helpful information, CCN strategy layer is able to set corresponding metrics in order to make optimized decision. The best source could be selected depend on link quality, optimal routes could be discovery based on transmission delay and reliability, node's neighbour information can also be used for suppressing hidden terminal caused collision. Hence, adopt Cross-Layer concept into CCN-VANET, can contributes to certain QoS covering aforementioned aspects.

\section{Conclusions}

In this work, we elaborate the challenges that confine the development of VANET then we argue the necessity to bring in ICN as an ideal solution for networking vehicular communication. A survey of recent related literatures is conducted from which we observed there are still research gaps in towards a seamless integration of CCN based vehicular communication. From there we proposed future research direction as well as positioning our succeeding works to make contribution to the field. 


\section{References}

[1] A. Bazzi, B. M. Masini, A. Zanella, C. D. Castro, C. Raffaelli, O. Andrisano, "Cellular aided vehicular named data networking”, 2014 International Conference on Connected Vehicles and Expo (ICCVE), pp. 747 - 752, Nov 2014.

[2] A.Iwata, C.-C. Chiang, G. Pei, M. Gerla, and T.-W. Chen, "Scalable Routing Strategies for Ad-hoc Wireless Networks,” In IEEE Journal on Selected Areas in Communications, Aug. 1999, pp. 1369-1379.

[3] B. Ahlgren, C. Dannewitz, C. Imbrenda, D. Kutscher, B. Ohlman, "A surey of information-centric networking", IEEE Communication Magazine, vol. 50, issue. 7, pp. 26-36, Jul 2012.

[4] C. D. Castro, C. Raffarlli, O. Andrisano, “A Dynamic Hierarhical VANET Architecture for Named Data Networking Applications”, 2015 IEEE International Conference on Communications (ICC), pp. 3659 - 3665, Jun 2015.

[5] D. Angermeier, A. Kiening, "PAL - privacy augmented LTE: a privacy-preserving scheme for vehicular LTE communication", VANET '13: Proceeding of the tenth ACM international workshop on Vehicular internetworking, systems, and applications, Jun 2013.

[6] D. Pesavento, G. Grassi, C. E. Palazzi, G. Pau. “A naming scheme to represent geographic areas in NDN", Wireless Days (WD), 2013 IFIP, pp. 1-3, Nov 2013.

[7] E. Lee, E. K. Lee, M. Geria, S. Y. Oh, "Vehicular Cloud Networking: Architecture and Design Principles”, IEEE Communications Magazine, vol .52, issue. 2, pp. 148155, Feb 2014.

[8] F. Bruno, M. Cesana, M. Gerla, G. Mauri, G. Verticale, "Optimal content placement in ICN vehicular networks”, Network of the Future (NOF), 2014 International Conference and Workshop on the, pp. 1-5, Dec 2014.

[9] F. D. da. Cunha, A. Boukerche, L. Villas, A. C. Viana, A, A. F. Loureiro. "Data Communication in VANETs: A Survey, Challenges and Applications”, [Research Report] RR-8498, INRIA Saclay. 2014. <hal00981126v3>.

[10] G. Deng, L. Shi, R. Li, X. Xie, “

Efficient Inter-Vehicle Internet Content Distribution Based on Named Data”, Vehicular Technology Conference (VTC Fall), 2015 IEEE 82 ${ }^{\text {nd }}$, Sep 2015.

[11] G. Deng, X. Xie, L. Shi, R. Li, "Hybrid information forwarding in VANETs through named data networking”, Personal, Indoor, and Mobile Radio Communications (PIMRC), 2015 IEEE 26th Annual International Symposium on, pp. 1940-1944, Aug 2015.

[12] G. Grassi, D.Pesavento, G. Pau, L. Zhang, S. Fdida, "Navigo: Interest Forwarding by Geolocations in Vehicular Named Data Networking”, Available at http://arxiv.org/pdf/1503.01713.pdf

[13] G. Grassi, D. Pesavento,G. Pau, R. Vuyyuru, R. Wakikawa, L. Zhang, "VANET via Named Data Networking”, Computer Communications Workshops
(INFOCOM WKSHPS), 2014 IEEE Conference on, pp. 410-415, Apr 2014.

[14] G. Mauri, M. Gerla, F. Bruno, M. Cesana, G. Verticale, "Optimal Contetn Prefetching in NDN Vehicle-toInfrastructure Scenario", Available at http://verticale.faculty.polimi.it/wpcontent/uploads/mobility.pdf

[15] "HIGH BANDWIDTH APPLICATION OVER WI-FI CONNECTION WITH IEEE 802.11p, AND IEEE P1609 (WAVE) PROTOCOLS”, Available: http://www.aradasystems.com/pdfs/Arada_IEEE_WAVE _paper.pdf

[16] K. Prasanth, K. Duraiswamy, K. Jayasudha, C. Chandrasekar, " Improved packet forwarding approach in vehicular ad hoc networks using RDGR algorithm”, International journal of next generation network(IJGN), vol. 2, no. 1, Mar 2010.

[17] K. C. Lee, U. Lee, M. Gerla, "Survey of routing protocols in vehicular ad hoc networks", Available at htttp://netlab.cs.ucla.edu/wiki/files/RoutingBookChapter KLULMario.pdf

[18] Lochert, C., Hartenstein, H., Tian, J., Fussler, H., Hermann, D., Mauve, M. (2003), "A routing strategy for vehicular ad hoc networks in city environments," Intelligent Vehicles Symposium, 2003. Proceedings. IEEE , vol., no., pp. 156-161, 9-11 June 2003.

[19] L. Chen, C. Englund, "Cooperative Intersection Management: A Survey", IEEE Transactions on Intelligent Transportation Systems, vol. 17, issue. 2, pp. 570-586, Feb 2016.

[20] L. C. Liu, D. Xie, S. Wang, Z. Zhang, "CCN-based cooperative caching in VANET", 2015 International Conference on Connected Vehicles and Expo (ICCVE), pp.198-203, Oct 2015.

[21] L. Wang, R. Wakilakiwa, R. Kuntz, R. Vuyyuru, L. Zhang, "Data naming in vehicle-to-vehile communications", Computer Communications Workshops (INFOCOM WKSHPS), 2012 IEEE Conference on, pp. 328-333, Mar 2012.

[22] L. Zhang, A. Afanasyev, V. Jacobson, K. Claffy, P. Crowley, C, Papadopoulos, L. Wang, B. Zhang, "Named Data Networking”, ACM SIGCOMM Computer Communication Review, vol. 44, no. 3, Jul 2014.

[23] L. Zhang, D. Estrin, J. Burke, V. Jacobson, J. D. Thornton, D. K. Smetters, B. Zhang, G. Tsudik, K. Claffy, D. Krioukov, D. Massey, C. Papadopoulos, T. Abdelzaher, L. Wang, P. Crowley, E. Yeh, "Named Data Networking (NDN) project” NDN, Technical Report NDN-0001, Oct 2010.

[24] M. Amadeo, C. Campolo, A. Molinaro, “Content-centric networking: is that a solution for upcoming vehicular networks?", VANET '12 Proceedings of the ninth ACM international workshop on Vehicular inter-networking, systems, and applications, pp. 99-102, Jun 2012.

[25] M. Amadeo, C. Campolo, A. Molinaro, "Design and analysis of a transport-level solution for content-centric VANETs”, 2013 IEEE International Conference on Communications Workshops (ICC), pp. 532 - 537, Jun 2013. 
[26] M. Amadeo, C. Campolo, A. Molinaro, "Forwarding strategies in named data wireless ad hoc networks: Design and evaluation”, Journal of Network and Computer Applications, vol. 50, issue. C, Apr 2015

[27] M. F. Al-Naday, A. Bontozoglou, V. G. Vassilakis, M. J. Reed, "Quality of service in an information-centric network", 2014 IEEE Global Communications Conference, pp. 1861-1866, Dec 2014.

[28] Named data networking, http://named-data.net

[29] N. Zorba, C. Skianis, C. Verikoukis, "Introduction: Why cross-layer? Its advantages and disadvantages," in Cross Layer Designs in WLAN Systems, United Kingdom, 2011.

[30] Perkins, C. E. and Royer, E. M. (1999), “Ad-Hoc OnDemand Distance Vector Routing,” Proc. IEEE WMCSA '99, New Orleans, LA, Feb. 1999, pp. 90-100.

[31] Q. Wang, D. Xie, X. Ji, "Network codes-based contentcentric transmission control in VANET", 2015 International Conference on Connected Vehicles and Expo (ICCVE), pp. 157-162, Oct 2015.

[32] R. A. Uzcategui, A. J. D. Sucre, G. A. Marum, "WAVE: a tutorial”, IEEE Communication Magazine, vol. 47, issue. 5, pp. 126-133, May 2009.

[33] R. Moalla,B. Lonc, H. Labiod, N. Simoni, “Towards a Cooperative ITS Vehicle Application Oriented Security Framework”, 2014 IEEE Intelligent Vehicles Symposium Proceedings, pp. 1043-1048, Jun 2014.

[34] S. H. Ahmed, S. H. Bouk, M. A. Yaqub, M, Gerla, "CONET: Controlled data packets propagation in vehicular Named Data Networks”, IEEE Consumer Communications and Networking Conference (CCNC), Jan 2016.

[35] S. H. Ahmed, S. H. Bouk, M. A. Yaqub, D. Kim, H. Song, J. Lloret, "CODIE: Controlled Data and Interest Evaluation in Vehicular Named Data Networks”, IEEE Transactions on Vehicular Technology, vol. 65, issue. 6, pp. 3954-3963, Apr 2016.

[36] S. H. Ahmed, M. A. Yaqub, S. H. Bouk, D. Kim, “ Towards content-centric traffic ticketing in VANETs: An application perspective”, 2015 Seventh International Conference on Ubiquitous and Future Networks, pp.237239, Jul 2015.

[37] S. Paul, J. Pan, R. Jain, "Architectures for the future networks and the next generation Internet: A survey", Computer Communications, vol. 34, issue. 7, pp. 2-42, Jan 2011.

[38] S. Rehman, M. A. Khan, T. Zia, L. Zheng, “ Vehicular ad-hoc networks(VANETs)-An overview and challenges" , EURASIP Journal on Wireless Communications and Networking 3(3):29-38, Jan2013.

[39] V. Kumar, S. Mishra, N. Chand, "Application of VANETs: Present \& Future”, Available at http://file.scirp.org/pdf/CN_2013110615180410.pdf

[40] V. Jacobson, “A Description of Content-Centric Networking (CCN)”, Future Internet Summer School (FISS), Bremen, Germany on July 22, 2009.

[41] V. Jacobson, D. K. Smetters, J. D. Thornton, M. F. Plass, N. H. Briggs, R. L. Braynard, "Networking named content”, CoNEXT '09 Proceedings of the 5th international conference on Emerging networking experiments and technologies, pp. 1-12, Dec 2009.

[42] W. Drira, F. Filali, “

A Pub/Sub extension to NDN for efficient data collection and dissemination in V2X networks”, World of Wireless, Mobile and Multimedia Networks (WoWMoM), 2014 IEEE 15th International Symposium on $a$, Jun 2014.

[43] Y. F. Chen, Z. T. Xiang, W. Jian, and W. Jiang. "A crosslayer AOMDV routing protocol for V2V communication in urban VANET”. Fifth International Conference on Mobile Ad Hoc and Sensor Networks, pp. 353-359, December 2009.

[44] Y. Li, T. Kanter, R. Rahmani, L. Wolf, “ An informationcentric approach for data dissemination in vehicular networks”, 2014 International Conference on Connected Vehicles and Expo (ICCVE), pp. 888-893. Nov 2014.

[45] Y. L. Morgan,“Notes on DSRC \& WAVE standards suite: its architecture, design and Characteristics ", IEEE Communication Surveys \& Tutorials, vol. 12, no. 4, 2010.

[46] Y. Rao, H. Zhou, D. Gao, H. Luo, Y. Liu, "Proactive Caching for Enhancing User-Side Mobility Support in Named Data Networking”, Innovative Mobile and Internet Services in Ubiquitous Computing (IMIS), 2013 Seventh International Conference on, pp. 37-42, Jul 2013.

[47] Y. Yu, X. Li, M. Y. Sanadidi, “ Scalable VANET content routing using hierarchical bloom filters”, 2013 9th International Wireless Communications and Mobile Computing Conference (IWCMC), pp. 1629-1623, Jul 2013.

[48] Y. Yu, Y. Li, X. Ma, W. Shang, M. Y. Sanadidi, M, Gerla. "Scalable opportunistic VANET Content Routing with encounter information”, 2013 21st IEEE International Conference on Network Protocols (ICNP), pp. 1-6, Otc2013.

[49] Y. Zhang, H. Zhang, L. Zhang, "Kite: A mobility support scheme for ndn”, NDN, Technical Report NDN-0020, 2014.

[50] Z. Yan, S. Zeadally, Y. Park, “ A Novel Vehicular Information Network Architecture Based on Named Data Networking (NDN)", IEEE Internet of Things Journal, vol. 1, issue. 6, pp. 525-532, Sep 2014.

[51] Z. Zhu, A. Alexander, L. Zhang, “A New Perspective on Mobility Support”, NDN, Technical Report NDN-0013, 2013. 